\title{
Audit of a tertiary heart failure outpatient service to assess compliance with NICE guidelines
}

\author{
Authors: Kaushik Guha, ${ }^{\text {A, }}$ Christopher ] Allen, ${ }^{\text {, }}$ Sumir Chawla, ${ }^{\mathrm{C}}$ Hayley Pryse-Hawkins, ${ }^{\mathrm{D}}$ Laura Fallon, ${ }^{\mathrm{D}}$ \\ Vicki Chambers, ${ }^{\mathrm{D}}$ Ali Vazir, ${ }^{\mathrm{E}}$ Alex R Lyon, ${ }^{\mathrm{E}}$ Martin R Cowie ${ }^{\mathrm{E}, \mathrm{F}}$ and Rakesh Sharma ${ }^{\mathrm{E}}$
}

The National Institute for Health and Care Excellence (NICE) updated its guidelines for chronic heart failure (HF) in 2010. This re-audit assessed interim improvement as compared with an audit in 2011. Patients with HF (preserved and reduced ejection fraction) attending a tertiary cardiac centre over a 2-year period (January 2013-December 2014) were audited. The data collected included demographics, HF aetiology, medications, clinical parameters and cardiac rehabilitation. In total, 513 patients were audited. Compared with 2011, male preponderance $(71 \%)$ and age $(68 \pm 14$ years, (Mean \pm SD)) were similar. $73 \%$ of patients lived outside of London. HF aetiologies included ischaemic heart disease ( $37 \%$ versus $40 \%$ in 2011 ), dilated cardiomyopathy ( $26 \%$ versus $20 \%$ ) primary valve disease ( $13 \%$ versus $12 \%$ ). For patients with left ventricular systolic dysfunction ( $n=434,85 \%$ of patients audited) $89 \%$ were taking beta-blockers (compared with $77 \%$ in 2011), $91 \%$ an angiotensin converting enzyme inhibitor or angiotensin receptor blocker ( $86 \%$ in 2011 ) and $56 \%$ a mineralocorticoid receptor antagonist ( $44 \%$ in 2011 ); $6 \%$ were prescribed ivabradine. All patients were reviewed at least 6-monthly. Although $100 \%$ of patients were educated about exercise, only 21 (4\%) enrolled in a supervised exercise programme. This audit demonstrated high rates of documentation, follow-up and compliance with guideline-based medical therapies. A consistent finding was poor access to cardiac rehabilitation.

KEYWORDS: Cardiac rehabilitation, heart failure management, left ventricular systolic dysfunction

Authors: ${ }^{\text {AST7 }}$ in cardiology, Cardiology Department, Royal Brompton Hospital, London, UK and National Heart and Lung Institute, Imperial College, London, UK; ${ }^{B}$ NIHR academic clinical fellow in cardiology, Cardiology Department, Royal Brompton Hospital, London, UK; C medical student, Cardiology Department, Royal Brompton Hospital, London, UK and University of

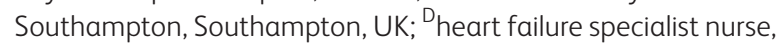
Cardiology Department, Royal Brompton Hospital, London, UK; Econsultant cardiologist, Cardiology Department, Royal Brompton Hospital, London, UK; F professor of cardiology, National Heart and Lung Institute, Imperial College, London, UK; " contributed equally

\section{Introduction}

Heart failure (HF) represents a major economic challenge to all developed countries and their allied health economies. It is a common condition with an increased prevalence with age. ${ }^{1}$ Patients are prone to repetitive prolonged, costly and potentially lethal hospitalisations. The economic burden for HF accounts for $2 \%$ of most westernised health economies; the same has previously been demonstrated within the NHS. ${ }^{2} \mathrm{HF}$ accounts for 1 million inpatient bed days (2\% of the NHS total) and 5\% of current emergency admissions to hospital, with $70 \%$ of the incurred costs being allied to hospitalisation. ${ }^{3,4}$ Notably, the prevalence of HF is predicted to further increase over the next decade because of an increasingly aging population. The pool of potential patients has also been expanded by the successes of strategies for treating other heart conditions, including primary percutaneous coronary intervention for ST-elevation myocardial infarction. ${ }^{5}$

HF may be subcategorised on the basis of cardiac imaging into populations with $\mathrm{HF}$ and preserved ejection fraction (HF$\mathrm{PEF}$ ), and HF with reduced ejection fraction (HF-REF) or left ventricular systolic dysfunction (LVSD). In terms of treatment strategy, there is now an established clear treatment regimen for patients with LVSD based on large randomised double blind controlled trials that have demonstrated the prognostic and clinical benefits of neurohormonal antagonism ${ }^{6-8}$ and beta adrenoceptor antagonists. ${ }^{9}$ Additionally for selected patients, further treatment options include cardiac resynchronisation therapy, implantable cardioverter defibrillators, cardiac transplantation and left ventricular assist devices. The evidence base for treatment within populations with HF-PEF has largely been disappointing and current guidance mandates the treatment of allied comorbidities with further trials ongoing within the area. ${ }^{10}$

The national ${ }^{3}$ and international guidelines ${ }^{11}$ summarise or reflect on the evidence base, with the most current recommendations from the National Institute for Health and Care Excellence (NICE) ${ }^{3}$ for chronic HF being published in 2010. Key priorities highlighted for implementation were as follows:

1 Ensure close monitoring of key clinical indices in patients with $\mathrm{HF}$ - functional status, fluid balance, heart rhythm, medication reviews and key biochemical parameters (eg renal profile). 
2 Increase prescription rates of established medical therapies in HF-REF/LVSD (beta-blockers, angiotensin converting enzyme inhibitors (ACEi)/angiotensin receptor blockers (ARB) and mineralocorticoid receptor antagonists (MRA).

3 Widen access to cardiac rehabilitation through group-based exercise programmes designed for patients with HF, which should include a component of psychological and educational support.

Here we delineate the completed audit cycle as compared to the current guidance within a tertiary hospital within an established professorial HF unit.

\section{The heart failure team}

The HF team at the Royal Brompton Hospital accepts national tertiary referrals for patients with HF of all aetiologies, serving a population spanning a large geographical area. With respect to outpatient management, the core team is composed of:

$>$ four specialist HF consultants (one professor and three consultants)

$>$ three full-time HF specialist nurses with hospital-based roles and a remit to liaise with a larger network of secondary care and community based-HF nurses

$>$ two deanery subspecialty training senior cardiology registrars, alongside a number of research and senior clinical fellows

$>$ dedicated cardiac pharmacists

deanery core medical trainees.

The HF team operates within the larger hospital network of consultant specialists in advanced cardiac imaging (echocardiography, cardiac magnetic resonance, nuclear, cardiac CT), cardiomyopathy, structural heart disease, interventional cardiology and cardiac surgery. A full palliative care team works alongside the HF team.

Outpatients with HF are served by seven consultant-led and four specialist nurse-led clinics with a weekly multidisciplinary team meeting for complex case discussion in parallel with further weekly forums for advanced device therapies and joint cardiology/cardiothoracic meetings for structural heart disease and revascularisation.

\section{Methods}

All HF patients attending Royal Brompton Hospital under the care of the HF team were included throughout two audit periods (2010-11 and 2013-14) following release of the NICE HF guidelines in $2010 .^{3}$ Patients with reduced or preserved ejection fraction were included, irrespective of their underlying aetiology. Outpatient reviews were audited for compliance with key indicators relating to three priorities for implementation described in the NICE guidelines. ${ }^{3}$ The first priority was patient monitoring, including assessment of clinical (functional capacity, fluid status, heart rhythm etc) and biochemical (serum urea, creatinine, electrolytes and estimated glomerular filtration rate (eGFR)) parameters at least 6-monthly in stable patients; second was prescription of evidence-based medication in HF-REF patients at maximum tolerated doses, specifically a beta-blocker and ACEi/ARB licensed for HF alongside and MRA if indicated; third was rehabilitation provision, including the education of all patients regarding the benefits of exercise in chronic HF and referral to an evidence-based rehabilitation programme.

The second audit period assessed improvement following the implementation of agreed action. Where appropriate, statistical analysis was performed using the Chi-Squared test, SPSS X7 (IBM, New York, USA). As the work completed represented a departmental audit, local ethical approval was not required.

\section{Agreed action implemented following first audit period}

Firstly, after an initial pilot period using a hard copy pro-forma began during the first audit period, an electronic data capture system was introduced covering the key monitoring indices outlined in the NICE guidelines. This system was extended to all HF clinics within the institution for the second audit period, enabling the complete capture of demographic data, HF aetiology, prescribed medication, heart rhythm and rate, measures of clinical follow-up (functional capacity, fluid status, renal profile etc) and compliance with rehabilitation. Secondly, after particular challenges with the provision of HF-based rehabilitation programmes were highlighted during the first audit period, further questions were added to better understand the process and highlight potential alternative solutions. Finally, a widespread education programme on evidencebased therapies in HF was introduced - targeted particularly at junior multidisciplinary team members in order to create more opportunities for appropriate treatment instigation and up-titration - alongside concerted engagement initiatives with GPs and referring secondary care trusts and patient education to provide ongoing senior support.

\section{Results}

\section{Clinical characteristics}

In total, 292 and 500 patients were included in the 2011 and 2014 audit cycles, respectively. There was a similar age profile $(67 \pm 14$ in $2011,68 \pm 142014, \mathrm{p}=0.33)$ and male preponderance $(70 \%, p=0.87)$ in both groups (Table 1). Compared with 2011, a greater proportion of the patients in the 2014 cohort were from outside of London $(74 \%$ versus $65 \%, \mathrm{p}<0.0001)$.

The predominant HF aetiologies were ischaemic heart disease (36\% (in 2011) versus 37\% (in 2014), $\mathrm{p}=0.81$ ), idiopathic dilated cardiomyopathy ( $19 \%$ versus $25 \%, \mathrm{p}=0.08)$ and primary valve disease ( $11 \%$ versus $13 \%, \mathrm{p}=0.49)$ (Table 1 ). In 2014, there was a significant increase in the proportion of patients with HF-PEF ( $8 \%$ versus $19 \%, \mathrm{p}<0.0001$ ). There were small numerical variances in the mean heart rate $(71 \pm 23$ in 2011 versus $68 \pm 12$ in $2014, \mathrm{p}=0.01$ ) and left ventricular ejection fraction ( $38 \pm 5$ in 2011 versus $40 \pm 13$ in 2014, $p=0.01$ ) of undetermined clinical significance.

\section{Monitoring}

Key clinical (functional capacity (89\%), fluid status (90\%), medication review $(87 \%)$, heart rhythm $(70 \%)$ and biochemical (eGFR (85\%), urea and electrolytes (78\%)) indicators were monitored at least 6 -monthly in a high proportion of patients during the initial audit cycle, and this subsequently increased significantly to near $100 \%$ completeness for all parameters upon re-audit in 2014 (Fig 1). Similarly, the proportion of 
Table 1. Demographics and clinical characteristics

\begin{tabular}{|c|c|c|c|}
\hline & $\begin{array}{l}2011 \text { cohort } \\
(n=292)\end{array}$ & $\begin{array}{l}2014 \text { cohort } \\
(n=500)\end{array}$ & p-value \\
\hline Age, years & $67 \pm 14$ & $68 \pm 14$ & 0.33 \\
\hline Male & $204(70)$ & $352(70)$ & 0.87 \\
\hline Resident in London & $128(35)$ & $132(26)$ & $<0.0001$ \\
\hline Heart rate, bpm & $71 \pm 23$ & $68 \pm 12$ & 0.01 \\
\hline LVSD & $268(92)$ & $404(81)$ & $<0.0001$ \\
\hline LVEF, \% & $38 \pm 5$ & $40 \pm 13$ & 0.01 \\
\hline \multicolumn{4}{|l|}{ Aetiology } \\
\hline Ischaemic & $105(36)$ & $185(37)$ & 0.81 \\
\hline $\begin{array}{l}\text { Idiopathic dilated } \\
\text { cardiomyopathy }\end{array}$ & 55 (19) & $122(25)$ & 0.08 \\
\hline Primary valvular & $32(11)$ & $64(13)$ & 0.49 \\
\hline Mixed & 29 (10) & $16(3)$ & 0.0002 \\
\hline Other & $51(17)$ & $81(16)$ & 0.69 \\
\hline Not documented & $20(7)$ & $32(6)$ & 0.88 \\
\hline
\end{tabular}

patients with a documented renal profile prior to initiation of an ACEi/ARB also increased (75\% in 2011 versus $100 \%$ in 2014, $\mathrm{p}<0.001$; Fig 1 ). By contrast, only a static small proportion of those commenced on an MRA did not have documented renal function (5\% in 2011, $5 \%$ in 2014, $\mathrm{p}=1.0$; Fig 1).

\section{Medication in left ventricular systolic dysfunction}

In comparison to the 2011 cohort, in 2014 the proportion of HF-REF patients prescribed the maximum tolerated dose of a beta-blocker ( $89 \%$ versus $77 \%$ in 2011, $\mathrm{p}=0.004$ ), ACEi/ARB ( $91 \%$ versus $86 \%$ in $2011, \mathrm{p}=0.002$ ) and MRA ( $56 \%$ versus $44 \%$ in 2011, $\mathrm{p}<0.001$ ) all increased significantly (Fig 2). In the 2014 cohort, $29(6 \%)$ patients were prescribed ivabradine.

\section{Rehabilitation}

Over the course of the audit period, there was a significant increase in the number of patients being educated about the benefits of exercise in chronic HF (36\% in 2011 to $100 \%$ in 2014, $\mathrm{p}<0.001$, Fig 3). Despite this, access to rehabilitation remained low, with only a small proportion being either referred $(6 \%$ in 2011 versus $3 \%$ in 2014, $\mathrm{p}=\mathrm{NS})$ or enrolled $(1 \%$ in 2011 versus $4 \%$ in 2014, $\mathrm{p}=\mathrm{NS}$ ) on a supervised exercise programme with an evidence base in HF.

\section{Discussion}

HF continues to pose serious challenges for healthcare systems and economies. The increasing prevalence of HF allied with prolonged and deadly hospitalisation ensures that it remains a significant and costly problem for global healthcare. Moreover, a significant proportion of hospitalisations that apparently occur as acute HF decompensation, transpire to be an acuteon-chronic decompensation.

The chronic care of patients with HF has been evaluated and summarised within national and international guidance. ${ }^{3,11}$ By rigorous and dedicated application/implementation, programmes following such guidance should reduce morbidity

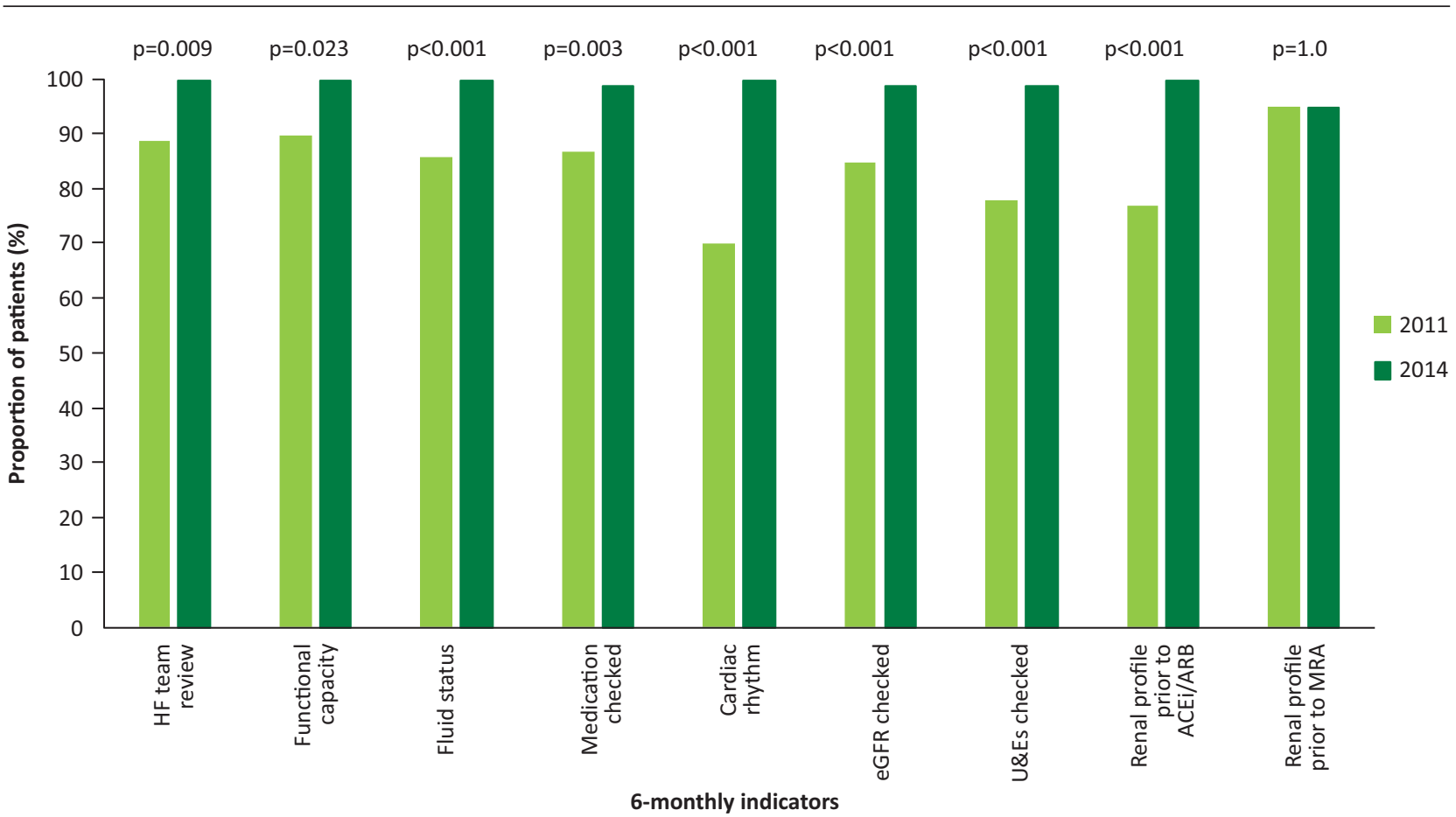

Fig 1. Monitoring: NICE 6-monthly indicators. The proportion of patients receiving the relevant monitoring at least 6-monthly. ACEi = angiotensin-converting-enzyme-inhibitor; $\mathrm{ARB}=$ angiotensin receptor blocker; eGFR = estimated glomerular filtration rate; $\mathrm{HF}=$ heart failure; $\mathrm{MRA}=$ mineralocorticoid receptor antagonist; NICE = National Institute for Health and Care Excellence; U\&E = urea and electrolytes. 


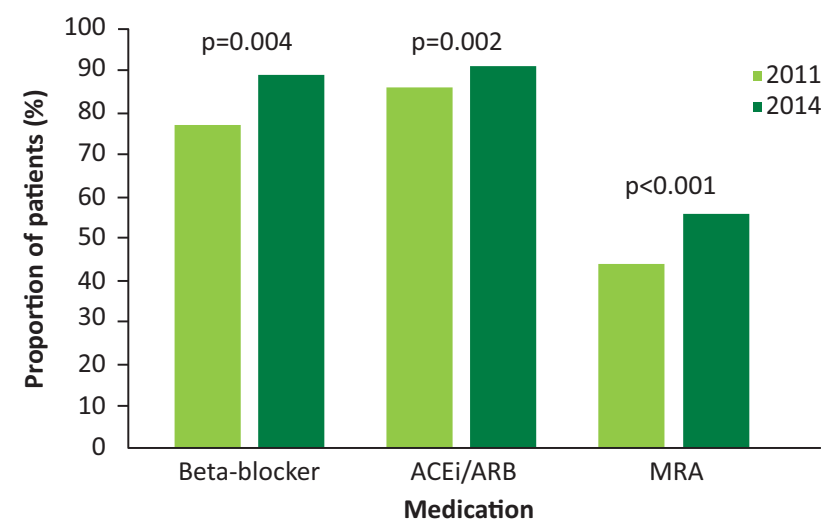

Fig 2. Left ventricular systolic dysfunction medication prescribing. The proportion of patients with left ventricular systolic dysfunction prescribed the relevant prognostic medical therapy. $\mathrm{ACE} i=$ angiotensin-converting-enzymeinhibitor; $\mathrm{ARB}=$ angiotensin receptor blocker; $\mathrm{MRA}=$ mineralocorticoid receptor antagonist.

and mortality within HF populations (with particular attention to those patients with LVSD). Emphasis is placed on lifestyle advice, appropriate usage of pharmacological evidence-based therapies, the selective application of advanced HF therapies (including cardiac resynchronisation therapy, implantable cardioverter defibrillators and assist devices, transplantation), disease monitoring and exercise-based rehabilitation. All of these aspects of care should be embedded within a multidisciplinary programme.

This completed audit focused on three critical areas for implementation highlighted in the NICE 2010 guideline $^{3}$ and, with an update currently under review, details progress made over the lifetime of this document within a professorial unit at a tertiary hospital in London. The rate of compliance with directives increased generally from 2011 to 2014 . The first key priority concerned monitoring, reflecting that care of HF patients mandates diligent follow-up with focus on symptomatic status, physical examination, pulse rate, rhythm, blood pressure and basic haematological and biochemical indices. Our audit demonstrates a clear improvement in

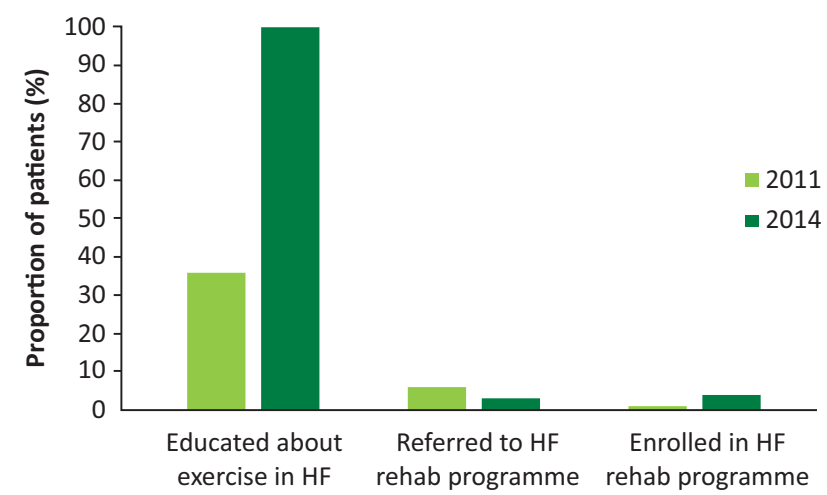

Fig 3. Access to cardiac rehabilitation. The proportion of patients with access to relevant cardiac rehabilitation. $\mathrm{HF}=$ heart failure. the monitoring of all of these components. The current recommendations suggest a monitoring interval of at least 6 months for those with stable chronic HF. Apart from the historical model of conventional healthcare within England and Wales, novel monitoring methods may be useful and may become the clinical norm in the future outpatient setting. This includes telemonitoring and implantable device-based remote monitoring. However, the currently available trial data reports conflicting conclusions, although further larger trials are expected to be reported in the future. ${ }^{12,13}$

For patients with LVSD, the rates of prescription for all recommended evidence-based pharmacotherapies significantly increased during the 4-year audit period (the second key priority) and now demonstrate high concordance with NICE standards. The prescription data from this audit is comparable to that obtained from large multicentre national and international registries. ${ }^{4,14,15}$ Ivabradine is a novel sino-atrial node inhibitor, which acts by selective inhibition of sodium channels within the sino-atrial node. This audit documents a rate of $6 \%$ usage within the LVSD population (29 patients). This reflects the updated technology appraisal guidance ${ }^{16}$ released in late 2012, which advocates ivabradine as a treatment option for controlling heart rate in certain HF populations.

The third priority for implementation concerned cardiac rehabilitation; an established part of the therapeutic intervention package in other areas of cardiology, including post myocardial infarction and revascularisation following coronary artery bypass grafting. However, within HF, cardiac rehabilitation has long been relatively neglected despite being recommended as an important part of the multidisciplinary treatment for patients. Rehabilitation-based programmes have previously been shown to improve both morbidity and mortality within such patients. ${ }^{17,18}$ They have also demonstrated clear benefits in improving rates of smoking cessation and reducing levels of anxiety and depression. ${ }^{19}$

Although the number of patients who had the benefits of regular cardiovascular exercise explained to them rose from $36 \%$ in 2011 to $100 \%$ in 2014 , the numbers actually referred to and accepted for rehabilitation still remain low. This is corroborated by national and European data, which demonstrate a consistently low level of enrolment of HF patients in rehabilitation programmes. ${ }^{19-21}$ Potential explanations for the disappointing levels of patient participation include geographical variation, lack of commissioning and financial restrictions. Hence, the current findings are suggestive that there needs to be a renewed and reinvigorated focus on the expansion and provision of rehabilitation services for HF patients, which should be highlighted as a key area for investment. Rather than using a conventional health facility-based rehabilitation programme, one alternative proposed for this group of possibly elderly and comorbid patients is to consider a home-based option instead. ${ }^{22}$ However, again there is limited evidence for such models in HF, with further exploratory work ongoing.

\section{Limitations}

This large audit cohort captures detailed data on the contemporary guideline-based management of chronic HF, although requires interpretation within certain limitations. 
The retrospective observational data from a single tertiary referral centre is subject to inherent selection bias and the complex multimorbid case mix the centre attracts may not be entirely generalisable. Furthermore, while high levels of data entry in the clinic database were observed, the prospect of some incomplete data influencing the conclusions cannot be entirely excluded. Finally, the audit design did not include standards in relation to compliance with recommendations for advanced device therapies (cardiac resynchronisation therapy and implantable cardiac defibrillators) outlined in the 2006 and 2014 NICE technology appraisals. ${ }^{23,24}$ The data collection process is being redesigned as a priority to cover this area for future work.

\section{Conclusion}

The audit cycle demonstrated an improvement in medical therapeutic prescription and increased adherence to monitoring recommendations achieved through simple agreed action. However, with regards to the provision of cardiac rehabilitation programmes for patients with HF, the available infrastructure still seems to be lacking and suggests that this aspect of care should be reviewed.

\section{Conflict of interest}

The authors declare no conflicts of interest.

\section{References}

1 Cowie MR, Wood DA, Coats AJ et al. Incidence and aetiology of heart failure; a population-based study. Eur Heart J 1999;20:421-8.

2 Pearson M, Cowie M (eds). Managing chronic heart failure: learning from best practice. London: Royal College of Physicians of London, 2005.

3 National Institute for Health and Care Excellence. Chronic heart failure in adults: management. NICE clinical guideline No 108. London: NICE, 2010.

4 Cleland J, Dargie H, Hardman S et al National Heart Failure Audit: April 2013-March 2013. London: National Institute for Cardiovascular Outcomes Research, 2013.

5 Townsend N, Wickramasinghe K, Bhatnagar P et al. Coronary heart disease statistics: a compendium of health statistics. London: British Heart Foundation, 2012.

6 Pitt B, Zannad F, Remme WJ et al. The effect of spironolactone on morbidity and mortality in patients with severe heart failure. $N$ Engl J Med 1999;341:709-17.

7 The Consensus Trial Study Group. Effects of enalapril on mortality in severe congestive heart failure. N Engl J Med 1987;316:1429-35.

8 The SOLVD Investigators. Effect of Enalapril on Survival in Patients with Reduced Left Ventricular Ejection Fractions and Congestive Heart Failure. N Engl J Med 1991;325:293-302.

9 CIBIS Investigators and Committees. A randomized trial of betablockade in heart failure. The Cardiac Insufficiency Bisoprolol Study (CIBIS). Circulation 1994;90:1765-73.
10 Lam CSP, Donal E, Kraigher-Krainer E, Vasan RS. Epidemiology and clinical course of heart failure with preserved ejection fraction. Eur J Heart Fail 2011;13:18-28.

11 McMurray JJV, Adamopoulos S, Anker SD et al. ESC guidelines for the diagnosis and treatment of acute and chronic heart failure 2012. Eur Heart J 2012;33:1787-847.

12 Chaudhry S, Mattera J, Curtis J et al. Telemonitoring in Patients with Heart Failure. N Engl J Med 2010;363:2301-9.

13 Morgan JM, Dimitrov BD, Gill J et al. Rationale and study design of the REM-HF study: remote management of heart failure using implanted devices and formalized follow-up procedures. Eur $J$ Heart Fail 2014;16:1039-45.

14 Maggioni AP, Anker SD, Dahlström U et al. Are hospitalized or ambulatory patients with heart failure treated in accordance with European Society of Cardiology guidelines? Evidence from 12440 patients of the ESC Heart Failure Long-Term Registry. Eur J Heart Fail 2013;15:1173-84.

15 Adams KFJr, Fonarow GC, Emerman CL et al. Characteristics and outcomes of patients hospitalized for heart failure in the United States: Rationale, design, and preliminary observations from the first 100,000 cases in the Acute Decompensated Heart Failure National Registry (ADHERE). Am Heart J 2005;149:209-16.

16 National Institute for Health and Care Excellence. Ivabradine for treating chronic heart failure. NICE technology appraisal guidance 267. Manchester: NICE, 2012.

17 Davies EJ, Moxham T, Rees $\mathrm{K}$ et al. Exercise training for systolic heart failure: Cochrane systematic review and meta-analysis. Eur $J$ Heart Fail 2010;12:706-15.

18 Piepoli MF, Davos C, Francis DP et al. Exercise training meta-analysis of trials in patients with chronic heart failure (ExTraMATCH). BMJ 2004;328:189.

19 British Heart Foundation. National audit of cardiac rehabilitation. Annual statistical report 2013. London: BHF, 2013.

20 Bjarnason-Wehrens B, McGee H, Zwisler A-D et al. Cardiac rehabilitation in Europe: results from the European Cardiac Rehabilitation Inventory Survey. Eur J Cardiovasc Prev Rehabil 2010;17:410-8.

21 Healthcare Commission. State of healthcare 2007: improvements and challenges in services in England and Wales. London: Healthcare Commission, 2007.

22 Clark RA, Conway A, Poulsen V et al. Alternative models of cardiac rehabilitation: a systematic review. Eur J Prev Cardiol 2015; 22:35-74.

23 National Institute for Health and Care Excellence. Implantable cardioverter defibrillators for arrhythmias. NICE technology appraisal 95. London: NICE, 2006.

24 National Institute for Health and Care Excellence. Implantable cardioverter defibrillators and cardiac resynchronisation therapy for arrhythmias and heart failure. NICE technology appraisal 314 London: NICE, 2014.

Address for correspondence: Dr Kaushik Guha, Department of Cardiology, Royal Brompton Hospital, Royal Brompton and Harefield NHS Foundation Trust, Sydney Street, London SW3 6NP, UK.

Email: k.guha@rbht.nhs.uk 